\title{
High Intensity Laser Physics at the Tata Institute
}

\author{
G. Ravindra KUMAR \\ Tata Institute of Fundamental Research, 1 Homi Bhabha Road, Mumbai 400 005, India
}

(Received June 19, 2010)

\begin{abstract}
This article presents the research and facilities in the area of high intensity laser physics at the Tata Institute of Fundamental Research in Mumbai, India. The Institute currently has a 20 terawatt (TW), 30 femtosecond (fs) table top chirped pulse amplification (CPA) titanium - sapphire laser facility and will have a fully functional $100 \mathrm{TW}, 25 \mathrm{fs}$ facility by the middle of 2011. This upgradation would propel existing research at the Institute to address ultraintense laser- matter interaction studies towards newer themes cutting across different areas of science - high energy density physics, physics of laser fusion, laboratory astrophysics, novel particle sources etc. It will also help in an even greater integration into the international community through joint experiments with visitors at the Institute.
\end{abstract}

Key Words: High energy density science, High intensity lasers, Laser produced plasmas, Hot electrons, Giant magnetic fields, Hard X-ray emission

\section{INTRODUCTION}

The Tata Institute of Fundamental Research (TIFR) was founded in 1946. It pursues basic sciences and mathematics, researching several sub-disciplines in these areas. Its main campus is located in Mumbai and it has several centers and facilities around India. The Institute has a long tradition of pursuing challenging problems and has set up big telescopes, ion accelerators, advanced fabrication facilities, state of the art imaging facilities etc. on its own, apart from playing an important role in international experiments abroad in high energy physics. The presence of researchers pursuing astrophysics, nuclear and atomic physics, particle physics, condensed matter physics, chemistry and biology in a highly interactive setting at one Institute, provides an excellent ground for the pursuit of high intensity laser physics with its multifaceted applications in basic science and advanced technologies. ${ }^{1)}$ Since the early 1990s the Tata Institute has pursued exploration of matter at high light intensities, and has made significant contributions. It now hopes to become a major player alongside the big groups in Europe, Japan and the American continent.

The interaction of intense $\left(>10^{16} \mathrm{~W} / \mathrm{cm}^{2}\right)$, femtosecond laser pulses with solid density plasmas not only provides interesting opportunities for the study of high energy density physics, but also facilitates the design of novel, micron sized, femtosecond x-ray, electron and ion sources. There is tremendous interest in such table-top pulsed X-ray sources for probing ultrafast real time dynamics in physical and chemical systems, micro-scale imaging, probing dense plasma, and lithography, to name a few of the multifaceted applications. Such exciting possibilities have led to an increasingly vigorous search for brighter sources and novel excitation schemes.

The Tata Institute has been probing basic physics related to laser light absorption and energy redistribution in various forms of matter - gaseous clusters, microdroplets, solid targets with polished and nanostructured surfaces etc. The group is also heavily interested in ultrafast time-resolved studies of the plasma with a view to understanding the dynamics of the laser interaction and plasma motion in real time. For details of the research over the past decade, please refer to www.tifr.res.in/ uphill (you can download many of our papers and theses)

\section{High Intensity Laser Facility at TIFR:}

High intensity laser research at TIFR received a boost in 2004 with the installation of a 7 terawatt (TW), 40 femtosecond (fs), $10 \mathrm{~Hz}$ repetition rate titanium-sapphire chirped pulse amplification (CPA) laser. The power of the laser was further boosted to $20 \mathrm{TW}$ at $30 \mathrm{fs}$ in 2006 accessing intensities exceeding $10^{19} \mathrm{~W} \mathrm{~cm}^{-2}$. In 2007 a project was approved for the installation of a laser with a power of $100 \mathrm{TW}$, by 2010 . This laser will now be commissioned in early 2011 and will be integrated into the experiments by middle of 2011.

I will now describe the laser systems before presenting some of the recent experimental results from the group. Figure 1 presents the outline of the $20 \mathrm{TW}$ laser system. The TIFR 20 terawatt laser is a typical Ti:Sapphire CPA system capable of delivering $600 \mathrm{~mJ}$ of $30 \mathrm{fsec}$ laser pulse at a repetition rate of $10 \mathrm{~Hz}$ with a spectral bandwidth of $35 \mathrm{~nm}$ at a central wavelength around $800 \mathrm{~nm}$. The laser consists of a Ti: Sapphire Oscillator (Femtosource) which provides $500 \mathrm{~mW}$, mode-locked femtosecond laser pulses with a bandwidth of about $52 \mathrm{~nm}$ at a repetition rate of $74 \mathrm{MHz}$. This laser is pumped by a solid-state, frequency doubled continuous $\mathrm{Nd}$ : $\mathrm{YVO}_{4}$ laser at $528 \mathrm{~nm}$ operated at a power level of $4 \mathrm{~W}$. In the CPA stage the oscillator pulse is stretched to $200 \mathrm{ps}$ to reduce the power of the pulse by an all-reflective stretcher and the stretched pulses are fed to a regenerative amplifier (regen) pumped by part of the energy produced by a frequency doubled pulsed Nd:YAG (total pulse energy $200 \mathrm{~mJ}$ at $532 \mathrm{~nm}$ ). An optical isolator before the regenerative amplifier ensures that no reflection is fed back into the oscillator to disturb mode-locking. After the regenerative amplifier, a pockels cell 
based 'pulse cleaner' minimizes the pre-pulse (post-pulse) which appear $8 \mathrm{nsec}$ before (after) the main pulse.

The pockels cell is activated electronically using a high voltage driver to ensure proper gain extraction from the amplifier. The output of the regenerative amplifier is sent to the first multi-pass (butterfly) amplifier, pumped by the remaining energy from the same pump laser. At the end of the regenerative amplifier the, titanium-sapphire laser pulse energy reaches up to a few $\mathrm{mJ}$ and after passing through the pre-amplifier the pulse energy is approximately $50 \mathrm{~mJ}$. This is further amplified in the next multi-pass amplifier stage pumped by two frequency doubled Nd:YAG lasers, each emitting pulses up to $1.5 \mathrm{~J}$ energy. This increases the stretched pulse energy to a final value of $1000 \mathrm{~mJ}$. After this the pulse is compressed back in time to $30 \mathrm{fs}$ with an energy of $600 \mathrm{~mJ}$ (after the transmission losses in the vacuum compressor) giving a peak power of 20 TW. The final beam has an approximately gaussian spatial profile with a diameter of $40 \mathrm{~mm}$. The nanosecond prepulse contrast, with respect to the main 30 femtosecond pulse is at the $10^{-5}$ level. The picosecond contrast is an order and a half poorer. Figure 2 shows some characteristics of the laser output.

At initial installation in 2004, the system produced a peak energy of $350 \mathrm{~mJ}$ per pulse in a $50 \mathrm{fs}$ pulse giving a peak power of $7 \mathrm{TW}$. The laser system has been quite amenable to modifications due to the modular nature of the subsystems. This peak power was boosted to the above mentioned levels by two modifications. (a) the introduction of a 'Dazzler' pulse shaper after the stretcher (to get a final pulse duration of 30 fs) and (b) the upgradation of the pump lasers of the final multi pass amplifier from $1 \mathrm{~J}$ at $532 \mathrm{~nm}$ to $1.5 \mathrm{~J}$ at $532 \mathrm{~nm}$. This increase in energy was obtained by changing to more efficient the second harmonic crystals in these lasers. The final titanium- sapphire laser pulse energy can be boosted up further from the present 1 Joule, if we add more pump lasers to the same amplifier configuration. A point to be noted is that a substantial reduction in cost was achieved by building the vacuum chamber for the compressor right in TIFR, indicating that high performance can be acquired at a reasonable cost.

\section{The TIFR 100 TW Ti-Sapphire CPA laser system}

This system which will be installed in early 2011, is not just a step towards higher intensities $\left(10^{21-22} \mathrm{~W} \mathrm{~cm}^{-2}\right)$ but also temporally much cleaner pulses. The broad architecture is that of a typical CPA laser (like the one described above), but with crucial improvements in pulse cleaning and pulse shaping. Briefly, the system architecture involves (a) a booster to in-

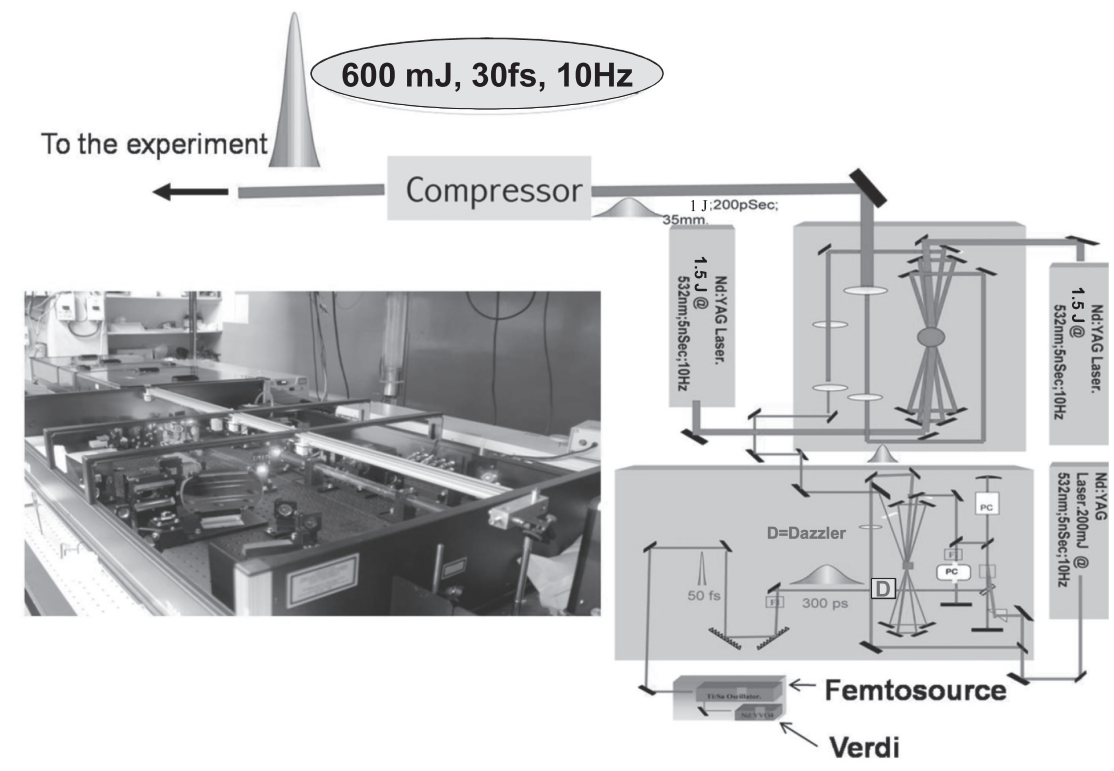

Fig. 1 (Right) Schematic diagram of the $20 \mathrm{TW}, 30$ fs laser at TIFR. Left: a part of the actual laser in operation.
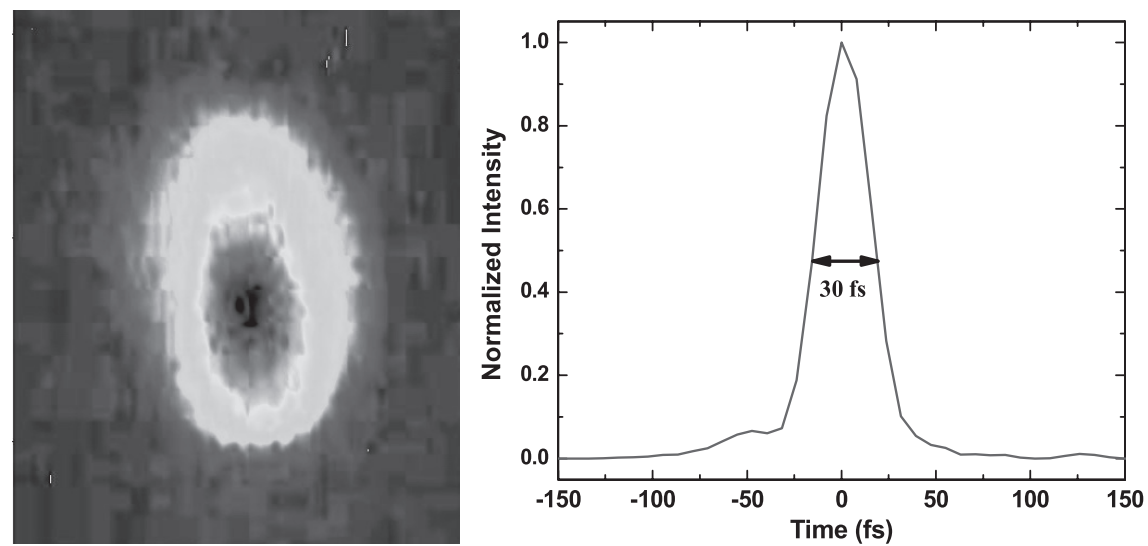

Fig. 2 Left: Spatial profile of the amplified beam before compressor. Right: Pulse duration. 


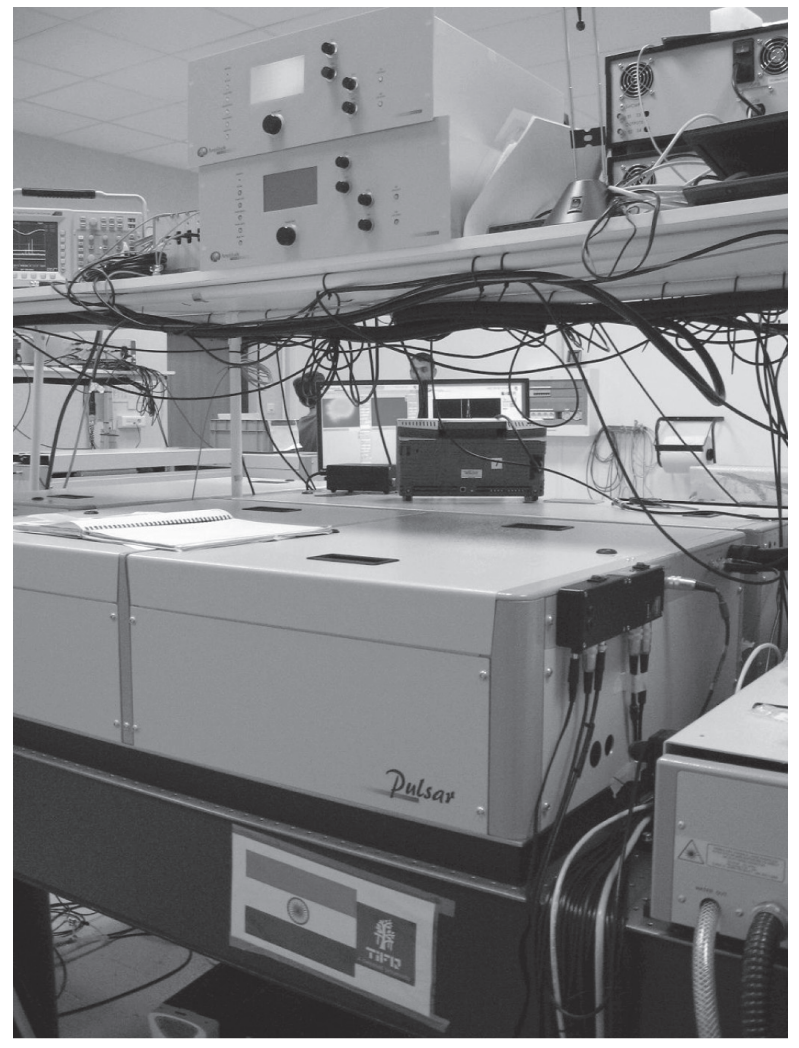

Fig. 3 The TIFR 100 TW laser system under construction.

crease the energy of the seed laser pulses to a microjoule level, (b) clean up of the temporal shape (picosecond scale) of this pulse by a saturable absorber, (c) stretching of the resultant pulse, (d) shaping of this by a 'Dazzler' and a 'Mazzler' (to fi-
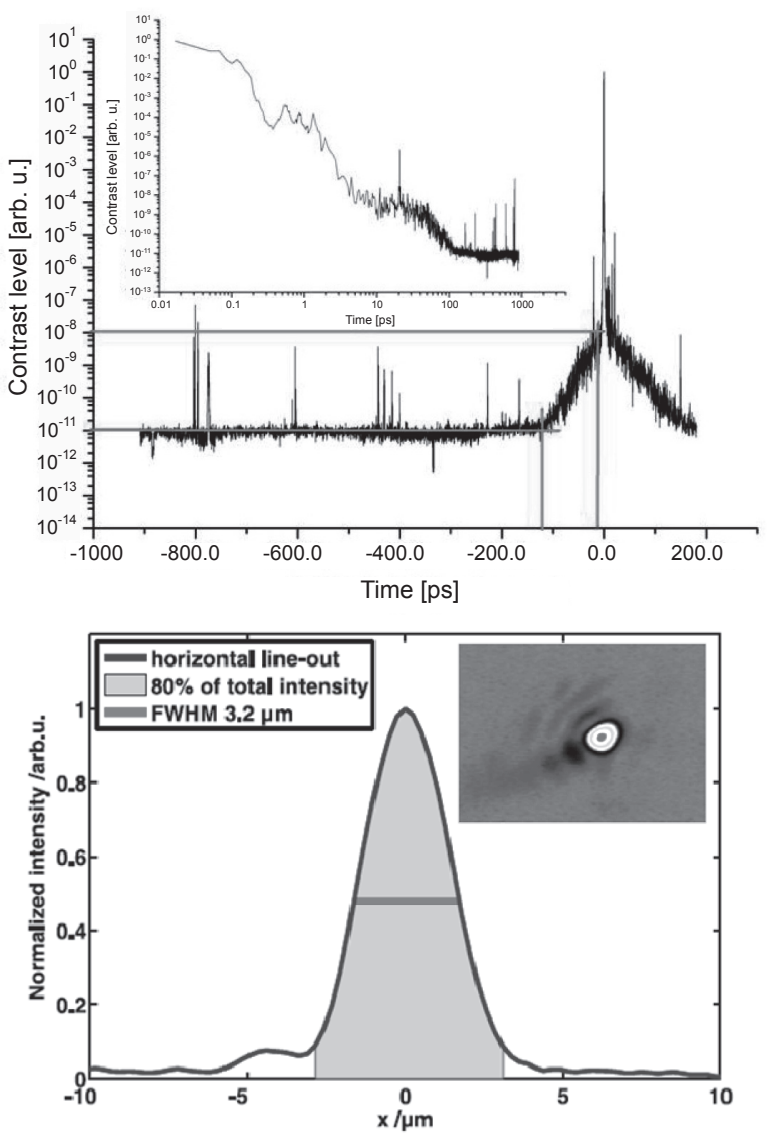

Fig. 4 Performance of the 100 TW laser system.

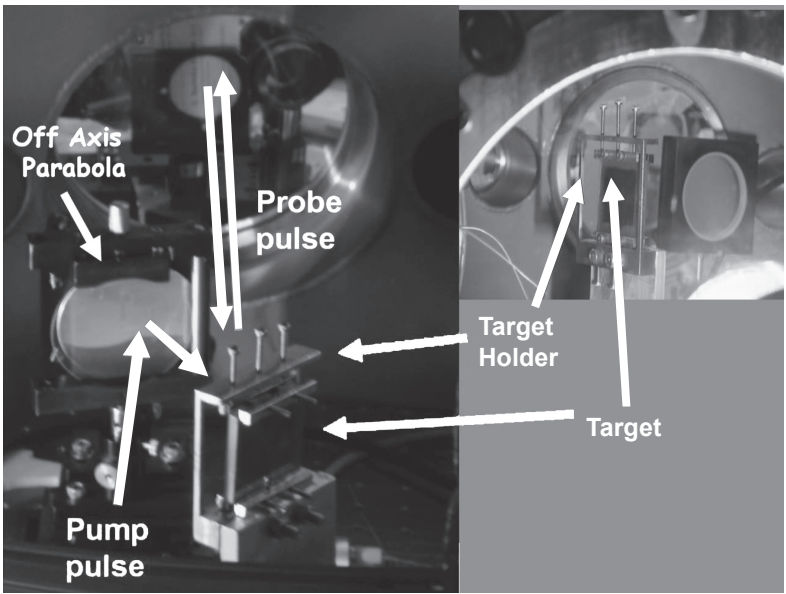

Fig. $5 \mathrm{~A}$ view of the inside of the target chamber: The off axis parabola focuses the pump laser beam onto the target. The probe pulse is derived from the compressor chamber and is independently controlled (wavelength, polarization) and is adjustably time delayed with respect to the pump beam.

nally obtain stable, 25-30 fs pulses), (e) amplification of this shaped pulses in a four stage amplifier and finally (f) compression of the amplified pulse back to 20-25 fs. The final amplifier is cryo-cooled to achieve an optimum and stable beam profile. ${ }^{2)}$ Pulse 'cleaning' is achieved by inserting Pockels cells at multiple locations. The nanosecond and picosecond

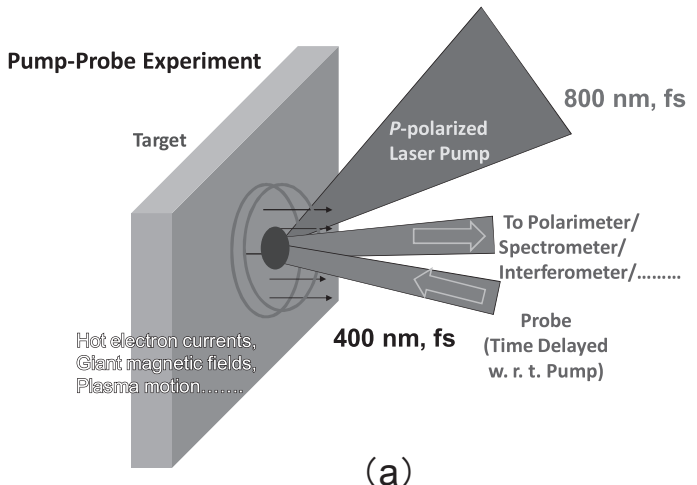

(a)

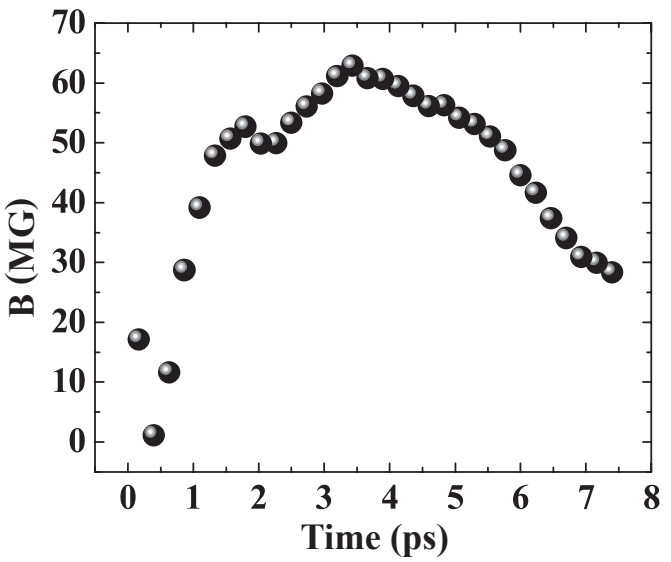

(b)

Fig. 6 Pump-probe studies of intense, femtosecond laser induced plasma; (a): Sketch of the set up. (b): Time evolution of the giant magnetic field induced in a solid density plasma at an intensity of $5 \times 10^{18} \mathrm{~W} \mathrm{~cm}^{-2}$. 


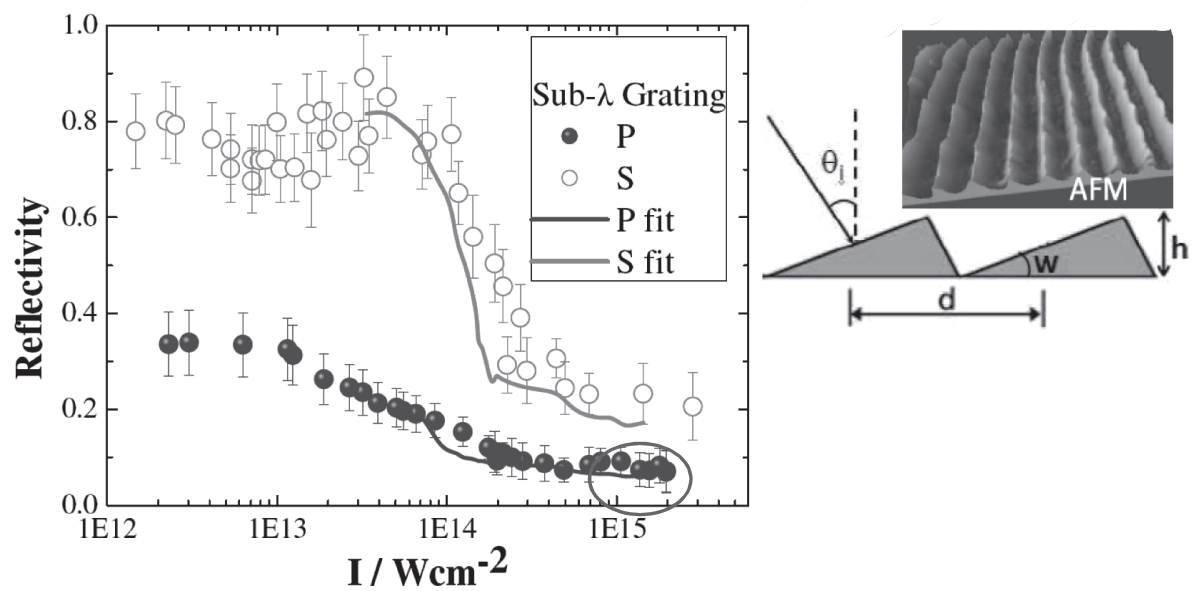

Fig. 7 Achieving nearly $100 \%$ intense laser absorption in a sub lambda grating target.

(a) Laser- $30 \mathrm{fs}$ $10^{19} \mathrm{~W} \mathrm{~cm}^{-2}$

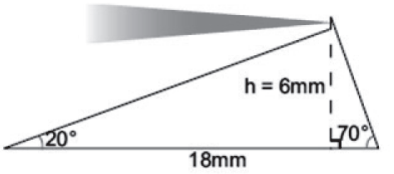

(b)

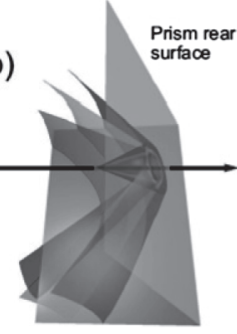

Frosted bottom surface

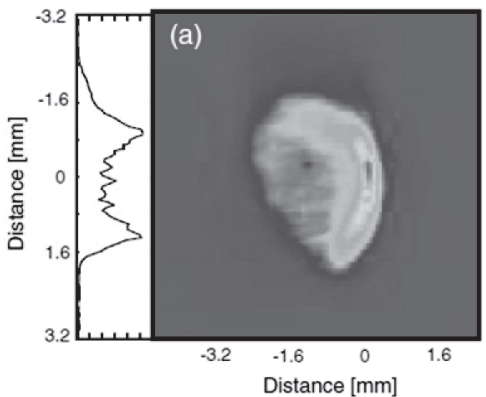

Spectrum of relativistic electrons

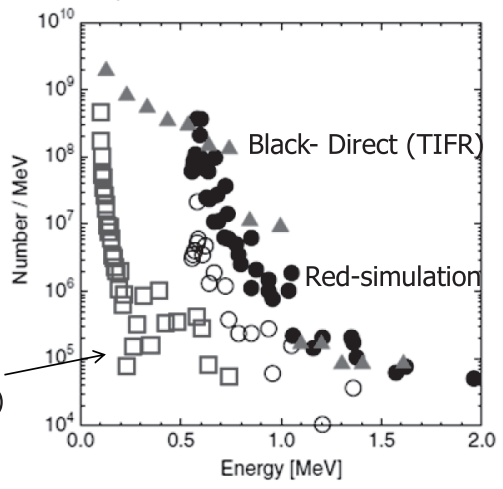

Fig. 8 Spectrally dispersed Cherenkov emission gives a direct measure of the relativivtic electron spectrum inside the target (Joint experiments with ILE, Osaka).

contrast levels are increased to levels of $10^{-11}-10^{-10}$, leading to clean femtosecond pulses. The cryo-cooling of the final amplifier has demonstrated good beam quality at the prescribed levels of $100 \mathrm{TW}$ and is expected to remain the same at higher levels of power that can be obtained by addition of more pump lasers. The pulse shaping counters the effect of gain narrowing and promotes reliability and reproducibility.

The laser system is being constructed by M/S Amplitude Technologies (France) in close consultation with TIFR. We have taken advantage of the experience of the other groups in this area to improve beam attenuation methods, monitoring of the pulse feedback into the system and refinement of the optical systems used in different parts. Figure 3 shows a part of the actual system, while Fig. 4 shows the typical performance achievable with such a laser.

\section{A flavour of research in high intensity laser science at TIFR:}

Though this article is mainly about the creation of high intensity laser pulses and user facilities, we will spend a brief while looking at some of the research possibilities, particular- ly with reference to the work at TIFR.

An overview of intense laser science and the Indian participation in this research has been covered in a recent review article. ${ }^{3)}$ Briefly, the main aims of the research at TIFR has been to look at the basic physics of the high intensity laser interaction with matter in different phases - solids, liquids (microdroplets), gaseous clusters of nanometre size etc. (studies on clusters and droplets are conducted by Prof. M. Krishnamurthy and his students). ${ }^{3)}$ In addition, several novelties have been attempted in terms of tailoring solid surfaces with nanostructures ${ }^{4)}$ and doping of gaseous clusters. ${ }^{5)}$ The aim of these attempts has been to enhance the generation of $\mathrm{keV}$ to $\mathrm{MeV}$ hot electrons and the resulting hard X-ray and ion emissions. Another crucial aspect of our efforts has been the study of the femtosecond dynamics of intense laser produced plasma. Special pump-probe set ups have been commissioned for this purpose.

Figure 5 shows the pump-probe set up in the target vaccum chamber used for solid targets in our laboratory.

Using such set ups, we have pioneered the study of the evolution of giant magnetic fields. ${ }^{6-9)}$ Figure 6 shows the sketch of the experimental set up and the temporal evolution of the 
magnetic fields in solid target plasma at an intensity of $5 \times 10^{18} \mathrm{~W} \mathrm{~cm}^{-2}$.

To give a flavour of some other recent work, I present two pictures below that reflect some of our major themes, namely hot electron generation and hot electron transport. In the first, intense laser absorption and hence hot electron generation is maximised by choosing a special target surface (a sub- lambda grating) while in the other, the transport of the hot electrons is studied, using their Cherenkov emission. I have chosen the later example to illustrate the links established between TIFR and labs in Japan. Figure 7 shows the enhanced absorption of 50 femtosecond laser pulses by invoking an extra absorption mechanism, namely the surface plasmon mode, excited at a particular angle of incidence (23 degrees) for a grating target with a spacing of $550 \mathrm{~nm}$. This spacing is smaller than the wavelength of the incident laser pulse $(800 \mathrm{~nm})$ and hence the grating becomes a 'sub- lambda' structure for the laser pulse. It turns out that this sub lambda structure facilitates nearly complete absorption of the incident laser pulse, leading to much hotter electron emission (increase of hot electron temperature from about $15 \mathrm{keV}$ to about $60 \mathrm{keV}$, i.e. by a factor of $\sim 4)$. Figure 8 shows an effective method to measure the energy of relativistically hot electrons created by the high intensity laser pulse. We achieve a direct, in situ measurement (hitherto not possible) by monitoring the Cherenkov radiation emitted by the relativistic electrons. This became possible by a major innovation in this experiment, namely a novel 'prism' target that dispersed the Cherenkov light in a manner that it provided one-to-one map between the energies of the relativistic electrons and their emission wavelengths.

In conclusion, I hope that I have given an idea of the aims of the high intensity physics research and some of the present and future facilities at the Tata Institute of Fundamental Research, Mumbai. High intensity laser physics research is also pursued majorly at two other institutions in India- RRCAT in Indore and BARC in Mumbai. For details, you may see the webpages (www.rrcat.gov.in and www.barc.gov.in) or refer to the review cited below.

\section{Acknowledgments}

I thank Hiromitsu Kiriyama for suggesting that I write this article and for his patience and persistence. It is a pleasure to acknowledge my TIFR colleagues, especially M. Krishnamurthy, P. Ayyub, J. Jha and A. D. Lad as well as several past students. The present lot of students - Sudipta Mondal, S. N. Ahmed, G. Chatterjee, P. Singh, R. Rajeev and postdoctoral fellow V. Narayanan are thanked for all their contributions over the past few years. We have benefitted a great deal on the theoretical aspects of plasmas from extensive and very fruitful collaboration with P. K. Kaw, S. Sengupta, Amita Das IPR, Gandhinagar. It is a pleasure to acknowledge our past and continuing association with K. A. Tanaka, H. Habara, R. Kodama and students (ILE, Osaka), T. Yabuuchi (now at UC, San Diego) and A.L. Lei, Z. M. Sheng, and W. M. Wang (China). We continue to learn from the research experience of R. Sauerbrey, U. Schramm, T. Cowan and colleagues at Forschungszentrum Rossendorf, Dresden (FZD) in the short pulse, high intensity arena and value their advice and collaboration. I acknowledge Amplitude Technologies, France for providing data on the laser system.

\section{References}

(www.tifr.res.in/uphill - provides a great deal of information on high intensity laser research at TIFR, which includes a list of papers and Ph. D. theses.)

1) G. Mourou, T. Tajima, and S.V. Bulanov: Rev. Mod. Phys. 78 (2006) 309.

2) G. Cheriaux: in Strong Laser Field Physics, (Ed: T. Brabec, Springer-Verlag, 2008).

3) G. Ravindra Kumar: Pramana- J. Physics 73 (2009) 113.

4) P. P. Rajeev, et al.: Phys. Rev. Lett. 90 (2003) 115002.

5) J. Jha and M. Krishnamurthy: Appl. Phys. Lett. 92 (2008) 191108; J. Jha and M. Krishnamurthy: J. Phys. B 41 (2008) 041002.

6) A. S. Sandhu, et al.: Phys. Rev. Lett. 89 (2002) 225002.

7) S. Kahaly, et al.: Phys. Plasmas 16 (2009) 043114.

8) S. Mondal, et al.: J.Phys. Conf. Series (To appear, 2010) (Proc. conference on "Inertial Fusion Science and Applications 2009").

9) G. Ravindra Kumar: J.Phys. Conf. Series (To appear, 2010) (Proc. conference on "Inertial Fusion Science and Applications 2009"Plenary Talk). 